\title{
Development of erythropoietin receptor-targeted drug delivery system against breast cancer using tamoxifen-loaded nanostructured lipid carriers
}

This article was published in the following Dove Press journal:

Drug Design, Development and Therapy

I4 March 2017

Number of times this article has been viewed

\author{
Chaw Yee Beh' \\ Chee Wun How ${ }^{1,2}$ \\ Jhi Biau Foo ${ }^{2}$ \\ Jia Ning Foong ${ }^{3}$ \\ Gayathri Thevi Selvarajah ${ }^{3}$ \\ Abdullah Rasedee ${ }^{1,3}$ \\ 'Laboratory of Vaccines and \\ Immunotherapeutics, Institute of \\ Bioscience, Universiti Putra Malaysia, \\ Serdang, ${ }^{2}$ Faculty of Pharmacy, MAHSA \\ University, Jenjarom, ${ }^{3}$ Faculty of \\ Veterinary Medicine, Universiti Putra \\ Malaysia, Serdang, Malaysia
}

\begin{abstract}
Tamoxifen (TAM) has been used in the treatment of breast cancers and is supplemented with erythropoietin (EPO) to alleviate the cancer-related anemia. The purported deleterious effects caused by the use of EPO with chemotherapeutic agents in the treatment of cancer-related anemia vary across studies and remain controversial. The use of nanoparticles as a drug delivery system has the potential to improve the specificity of anticancer drugs. In this study, we simultaneously incorporated two pharmacological active ingredients in one nanocarrier to develop EPO-conjugated TAM-loaded lipid nanoparticles (EPO-TAMNLC), a targeted delivery system, to enhance the cytotoxic activity while reducing the side effects of the ingredients. The effect of temperature in modulating the thermodynamic parameters associated with the binding of EPO and TAMNLC was assessed using isothermal titration calorimetry, while the unfolding of EPO structure was determined using fluorescence-quenching approach. The association efficiency of EPO and TAMNLC was $55.43 \%$. Unlike binding of albumin to TAMNLC, the binding of EPO to TAMNLC occurred through endothermic and entropy-driven reaction. The EPO-TAMNLC formulation was stable because of the hydrophobic interaction and the high free energy, suggesting the spontaneity of the interactions between EPO and TAMNLC. The EPO-TAMNLC enhanced the in vitro cytotoxicity of TAM to MCF-7 cells. The EPO surface-functionalized TAMNLC could sequentially deliver EPO and TAM as well as improving site-specific delivery of these therapeutic compounds.
\end{abstract}

Keywords: tamoxifen, thermodynamic interaction, albumin

\section{Introduction}

Breast cancer is a disease caused by abnormal cell division that occurs commonly in the lining of milk ducts and lobules. Despite the availability of various diagnosis and treatment procedures, the disease can be worsen through metastasis. Current therapeutic regimes for cancers are fraught with varying degree of effectiveness and debilitating side effects. Cancer treatments target specific cell signaling pathways with the ultimate aim of causing cancer cell death. ${ }^{1}$ Due to the lack of specificity of current chemotherapeutic drugs, the bone marrow production of blood cells is often compromised, resulting in anemia in $>75 \%$ of treated cancer patients. ${ }^{2}$ This will burden the anemia of chronic diseases that often manifest in cancer patients. ${ }^{3}$ Anemia impairs the efficacy of cancer therapies, deteriorates quality of life, and is fatal if untreated.

Erythropoietin (EPO), a $34 \mathrm{kDa}$ glycoprotein produced mainly in the kidneys, is prescribed for the treatment of anemia. Although beneficial to anemic patients
Correspondence: Abdullah Rasedee Laboratory of Vaccines and Immunotherapeutics, Institute of Bioscience, Universiti Putra Malaysia, 43400 UPM Serdang, Selangor, Malaysia Tel +60389473455

Email rasedee@upm.edu.my
Drug Design, Development and Therapy 2017:II 77I-782

771 
through stimulation of hematopoiesis, the use of EPO for cancer-related anemia is being reviewed because of the higher mortality rate in breast cancer patients treated with the hormone. ${ }^{4}$ In breast cancer treatment, EPO can alleviate anemia symptoms, but may also exert effect in the breast tissues. This is due to the fact that numerous cancer cells including the breast cancers have been shown to contain functional erythropoietin receptor (EpoR), and these receptors play an important role in the proliferation and migration of these cells. ${ }^{5-7}$

Tamoxifen (TAM) is one of the drugs approved by the US Food and Drug Administration for the treatment and prevention of breast cancers. It seems that the EPO and TAM combination treatment resulted in a $90 \%$ breast tumor regression in mammary tumor-induced Sprague Dawley rats, outperforming the efficiency of TAM alone which was only $70 \% .^{8}$ These findings suggest that EpoR in breast tissues, especially the breast tumors, can be exploited as targets in cancer therapy using EPO as homing ligands.

The paradigm of using nanoparticulate system as pharmaceutical carriers to enhance the efficacy of anticancer compounds has now been well documented. Among the various nanoparticulate drug delivery systems, the nanostructured lipid carriers (NLCs) are the most extensively studied because they meet the requirements of an ideal carrier system for lipophilic compounds. Their low cytotoxicity, capability to protect drug from degradation, high drug payload, and passive-targeting ability are compelling attributes that promote them as carriers of anticancer drugs. ${ }^{9}$ In addition, the possibility of scale-up production makes NLC an attractive drug carrier system for commercial applications. Numerous active pharmaceutical ingredients (APIs), including TAM, had been formulated with NLC while developing as drug delivery carriers. ${ }^{10-12}$ The tamoxifen-loaded nanostructured lipid carrier (TAMNLC) with an average size of $\sim 50 \mathrm{~nm}$ exhibits a first-order drug-release kinetics. ${ }^{12}$ The cytotoxic effect of TAM remains relatively unchanged after formulation with the NLC.

Previous studies have shown that modification of nanoparticles surface can enhance their binding of compounds, targeting, stability, and activity. ${ }^{13}$ In our study, the main objective is to develop a surface-modified TAM-entrapped NLC with EPO that could be delivered in a sequential manner to the target tissue. Following the development of stable TAMNLCs, we modified the surface of these drug nanocarriers to develop the EPO-TAMNLC. The interactions of EPO and albumin in NLC were investigated using isothermal titration calorimetry (ITC) to determine the thermodynamic forces involved. ${ }^{14}$
The combined delivery of EPO and TAM using a single carrier is postulated to facilitate their synergistic effect, as they are released and delivered in a sequential manner, thereby enhancing the specificity of the EPO-TAM combination.

\section{Materials and methods Materials}

Hydrogenated palm oil or Softisan 154 (Condea Chemie, Hamburg, Germany) was donated by Malaysia Palm Oil Board (MPOB), and olive oil was purchased from Basso Fegele and Figli Srl (San Michele di Serino, Italy). Other ingredients used were Lipoid S100 (Lipoid GmbH, Ludwigshafen, Switzerland), polysorbate 80 (Thermo Fisher Scientific, Waltham, MA, USA), thimerosal, and sorbitol (Sigma-Aldrich, St Louis, MO, USA).

Bovine serum albumin (BSA), dimethyl sulfoxide (DMSO), (4-(2-hydroxyethyl)-1-piperazineethanesulfonic acid) (HEPES), 2-(N-morpholino)ethanesulfonic acid (MES) sodium salt, uranyl acetate, sodium acetate, sodium iodide, TAM free-base, and thiazolyl blue tetrazolium bromide (MTT) were purchased from Sigma-Aldrich. Sodium dodecyl sulfate polyacrylamide gel electrophoresis (SDS-PAGE) reagent starter kit (\#1615100) was purchased from Bio-Rad Laboratories (Hercules, CA, USA). Other chemicals used were Eprex 10,000 IU (Janssen-Cilag Ltd., Beerse, Belgium), methanol, and acetic acid (Thermo Fisher Scientific). Ultrapurified water was dispensed from a Milli-Q Plus water purification system (Merck Millipore, Billerica, MA, USA).

\section{Tamoxifen-loaded nanostructured lipid carrier}

TAMNLC was prepared using lipid melt-emulsification technique. ${ }^{15}$ Briefly, a mixture of hydrogenated palm oil $(4 \%, \mathrm{w} / \mathrm{v})$, olive oil $(1 \%, \mathrm{v} / \mathrm{v})$, lecithin $(1.73 \%, \mathrm{w} / \mathrm{v})$, and TAM (200 mg) was heated to about $70^{\circ} \mathrm{C}$. Then, a hot aqueous phase containing sorbitol $(4.75 \%, \mathrm{w} / \mathrm{v})$, polysorbate 80 $(1 \%, \mathrm{v} / \mathrm{v})$, and thimerosal $(0.005 \%, \mathrm{w} / \mathrm{v})$ was added. The hot emulsion was homogenized at 13,000 rpm for 10 min using a high-sheer homogenizer (IKA, Staufen, Germany), followed by high-pressure homogenization at 1,000 bar for 20 cycles using Emulsiflex C-50 (Avestin, Ottawa, ON, Canada). Then, the hot emulsion was cooled to room temperature to form TAMNLC and kept for 2 days before characterization. The particle size, zeta potential, and polydispersity index were determined using Zetasizer Nano ZS (Malvern, UK), while the entrapment efficiency was confirmed using an Alliance Separation Module HPLC (Waters, Milford, MA, USA). 
For more details on how the measurements were presented, please see Supplementary materials S1 and $\underline{\mathrm{S} 2}$.

\section{Transmission electron microscopy}

Transmission electron microscopy was used to visualize the morphology of the nanoparticles. A drop of diluted EPOTAMNLC sample was placed on the surface of a carboncoated copper grid (300 mesh size). The droplet was removed with a filter paper after $1 \mathrm{~min}$. Then, the grid was stained with uranyl acetate for $3 \mathrm{~min}$ and allowed to get air-dried at room temperature. The EPO-TAMNLC sample was probed using a transmission electron microscope (Hitachi H-7100; Hitachi Ltd., Tokyo, Japan), and the images were captured (Figure 1).

\section{Fluorescence-quenching assay}

The folding of protein could affect its association to an interface. Therefore, the folding of EPO and BSA was examined under different conditions. Fluorescence-quenching assay of EPO and BSA was performed using NaI. The tryptophan (Trp) subunits in the proteins were examined at an excitation wavelength of $295 \mathrm{~nm}$, and an emission wavelength in the range of $310-400 \mathrm{~nm}$ with $2 \mathrm{~nm}$ increments. Eighty five microliters of $87 \mu \mathrm{g} \mathrm{mL}^{-1}$ protein sample was dispensed into a flat-bottomed black multiplate (Nunc Nalgene, Penfield, NY, USA) and incubated for $1 \mathrm{~h}$ with $115 \mu \mathrm{L}$ of four different buffers $(10 \mathrm{mM})$ respectively. Then, the protein samples were quenched with $5 \mu \mathrm{L}$ of $5 \mathrm{M} \mathrm{NaI}$. The dilution effect due

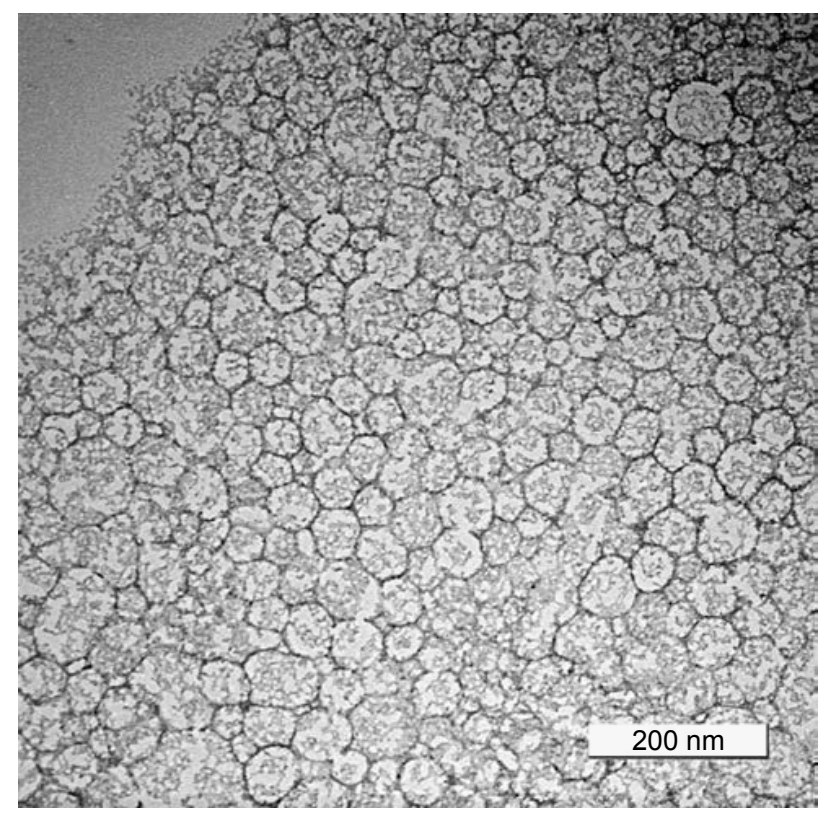

Figure I Transmission electron microscope image (20,000x magnification) of erythropoietin-conjugated tamoxifen-loaded nanostructured lipid carrier. to the addition of quencher was minimized by reducing the volume of the quencher. To determine the effect of $\mathrm{pH}$ on the quenching efficiency (QE) of NaI, buffers of various $\mathrm{pHs}$ were used: ${ }^{16}$ sodium acetate $(\mathrm{pH} 5,5.5)$, MES ( $\left.\mathrm{pH} 6,6.5\right)$, HEPES ( $\mathrm{pH} 7,7.5)$, and Tris- $\mathrm{HCl}(\mathrm{pH} 8,8.5)$. The conductivity of the buffer systems was normalized to $4.5 \mathrm{mS} \mathrm{cm}^{-1}$ using a $4 \mathrm{M} \mathrm{NaCl}$ solution to prevent interference.

The sample plates were examined using a Synergy H1 fluorescence mode reader (Biotek, Winooski, VT, USA) at $25^{\circ} \mathrm{C}$ and analyzed with Gen5 software. The experiment was performed in triplicates. The QE was calculated and the SternVolmer plot was generated using the following equations:

$$
\begin{gathered}
\mathrm{QE}=\frac{F_{0}-F}{F_{0}} \\
\frac{F_{0}}{F}=1+\mathrm{K}_{\mathrm{SV}}[\mathrm{Q}]
\end{gathered}
$$

where

$F_{0}=$ fluorescence emission before addition of quencher

$F=$ fluorescence emission after addition of quencher

$\mathrm{K}_{\mathrm{SV}}=$ Stern-Volmer constant

$[\mathrm{Q}]=$ concentration of quencher

\section{Protein-TAMNLC association efficiency and thermodynamic interactions}

The amount of EPO absorbed onto TAMNLC was determined using SDS-PAGE. ${ }^{17}$ The proteins and TAMNLC at a 1:1 ratio were incubated overnight in buffers of various pHs before electrophoresis. For electrophoresis of BSATAMNLC and EPO-TAMNLC, $8 \%$ and $12 \%$ acrylamide gels were casted, respectively. Then, they were stained with Coomasie blue (Sigma-Aldrich), followed by an overnight de-staining procedure using methanol, water, and acetic acid $(50: 5: 45, \mathrm{v} / \mathrm{v} / \mathrm{v})$. The intensity of the protein bands was determined using a ChemiDoc MP Imaging System (Supplementary materials).

The thermodynamic interaction of EPO and BSA with TAMNLC was investigated using a Nano-ITC (TA Instruments, New Castle, DE, USA). All TAMNLC samples were dialyzed with excess phosphate buffer saline (PBS) (Supplementary materials). The binding of the proteins to the nanocarriers at various temperatures was determined after 25 injections of $2 \mu \mathrm{L}$ aliquot of $15 \mu \mathrm{M}$ proteins into the sample cell containing $300 \mu \mathrm{L}$ TAMNLC suspension. Control experiments were performed by titrating the proteins into buffers without TAMNLC. The corrected heat data were 
analyzed using NanoAnalyze software (V3.3.0) with an independent binding model (Supplementary materials).

\section{Cell receptor status and functional study of EPO-TAMNLC}

The estrogen receptor- $\alpha(E R \alpha)$ and EpoR status of MCF-7 and MDA-MB231 cell lines was confirmed via immunocytochemical staining (Supplementary materials). The MDA-MB231 cell line, derived from triple-negative breast cancer, was used as the negative control. The cell lines were cultured in a six-well plate (Nunc Nalgene) with coverslip. The cells were rinsed with PBS and fixed with $4 \%(\mathrm{w} / \mathrm{v})$ paraformaldehyde (Thermo Fisher Scientific). The fixed cells were rinsed with PBS before they were permeabilized using $0.1 \%(\mathrm{w} / \mathrm{v})$ Triton-X 100 solution. Subsequently, the cells were blocked by incubating them with $1 \%(\mathrm{w} / \mathrm{v}) \mathrm{BSA}$ solution for $1 \mathrm{~h}$. The ER $\alpha$ antibody (sc542-AF488) (Santa Cruz Biotechnology, Dallas, TX, USA) and EpoR antibody (sc697) (Santa Cruz Biotechnology) were incubated with the cells for $2 \mathrm{~h}$ at room temperature and overnight at $4{ }^{\circ} \mathrm{C}$, respectively. Anti-rabbit antibody (sc3739) tagged with phytoerythrin was added as the secondary antibody. The cells were counterstained with 4',6-diamidino-2-phenylindole (DAPI). The blue nuclei, green ER $\alpha$, and red EpoR were visualized with IX73 Live Cell Imaging fluorescent microscope (Olympus, Tokyo, Japan).

The cytotoxic activity of EPO-TAMNLC was tested on $\mathrm{HDFa}$ (primary human dermal fibroblast normal human adult, ATCC PCS-201-012) and MCF-7 cell line (mammary adenocarcinoma human cell line, ATCC HTB-22) via MTT assay. At 24, 48, and $72 \mathrm{~h}$, the concentration-response curves of the treatments were generated, while their respective cytotoxicity response parameters were determined. Specifically, the halfgrowth inhibitory concentration $\left(\mathrm{GI}_{50}\right)$, total growth inhibition concentration, and lethal concentration $50\left(\mathrm{LC}_{50}\right)$ were acquired (Supplementary materials).

\section{Statistical analysis}

The data obtained were statistically analyzed, and the results expressed as mean \pm standard deviation. One-way analysis of variance was performed using IBM SPSS version 20 software.

\section{Results}

The fine dispersion of the lipid nanoparticles was opalescent in appearance. The size ( $Z$-average), zeta potential, polydispersity index, and entrapment efficiency of the TAMNLC were $59.36 \pm 0.25 \mathrm{~nm},-7.20 \pm 1.80 \mathrm{mV}, 0.24 \pm 0.01$, and
$99 \%$, respectively. Once EPO was conjugated to TAMNLC (EPO-TAMNLC), the size, zeta potential, and polydispersity index were $55.39 \pm 0.98 \mathrm{~nm},-1.58 \pm 0.47 \mathrm{mV}$, and $0.19 \pm 0.01$, respectively. Figure 1 shows the electron micrograph of EPOTAMNLC. The particles in EPO-TAMNLC were in submicron range and appeared in aggregated spherical shape.

The EPO and BSA folding was examined using their intrinsic fluorescence caused by the hydrophobic amino acid residues present within them (ie, Trp). ${ }^{18}$ Upon exposure to the external environment, the Trp fluorescence was quenched by negatively charged iodide (Figure 2). As the $\mathrm{pH}$ of the medium increased from 5.0 to 8.0, QE decreased. At acidic pH, BSA had more Trp residues exposed that were quenched by the iodide. At alkaline $\mathrm{pH}$ however, the conformation of BSA became less distorted, shielding the hydrophobic Trp from the quenching effect of iodide. Iodide was present in buffers used as the control.

The intrinsic fluorescence of EPO was caused by its three Trp residues. There were no dramatic changes in the emission maxima wavelength $\left(\lambda_{\max }\right)$ of EPO in the $\mathrm{pH}$ range of 5.0-8.5, although a higher quenching effect was observed at $\mathrm{pH}$ 5. No significant difference was seen at $\mathrm{pH}$ 6.5-8.0. Thus, we hypothesized that a $\mathrm{pH}$ lower than the physiological value may trigger an increase in solvent exposure of nonpolar region of the EPO that may have promoted binding to hydrophobic surfaces of the NLC. In addition, at the physiological $\mathrm{pH}$ of $\sim 7.0$, there was quenching of EPO, suggesting that the exposure of Trp can occur in the natural environment of the circulation.

At steady state, where $\mathrm{pH}$ ranged from 5.0 to 8.5 , the $\lambda_{\text {max }}$ of EPO was $\sim 336 \mathrm{~nm}$ (data not shown). This suggested any $\mathrm{pH}$-induced change in EPO structure is subtle which does not affect the intrinsic fluorescence of the protein. The subtle change in EPO was shown by its sensitivity to iodide in low-pH aqueous environment that may be the result

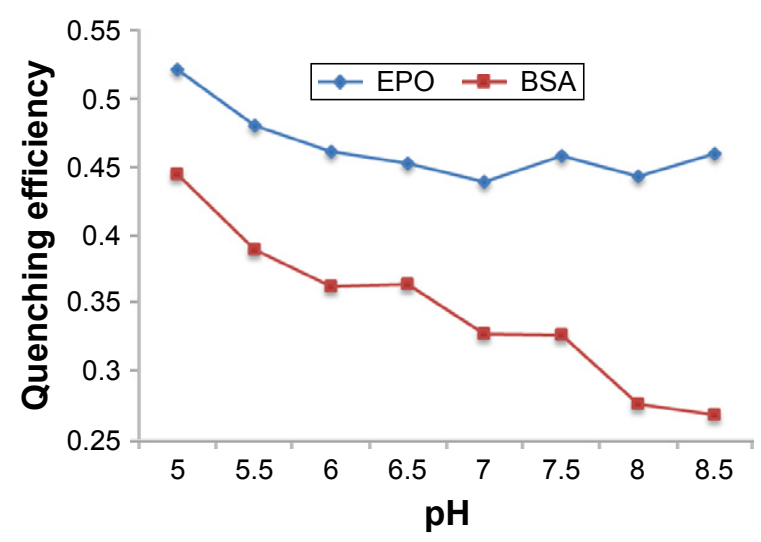

Figure 2 Influence of $\mathrm{pH}$ on quenching efficiency of iodide for EPO and BSA. Abbreviations: EPO, erythropoietin; BSA, bovine serum albumin. 


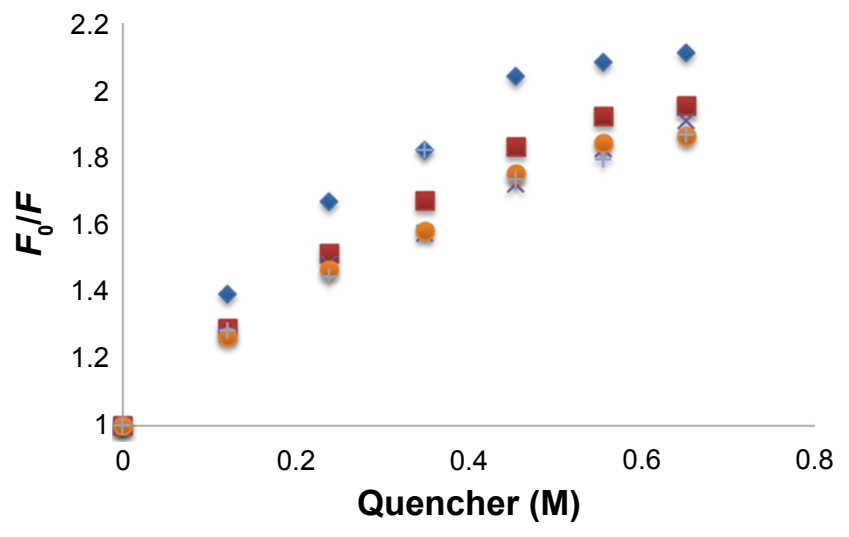

pH $5.0 \square \mathrm{pH} 5.5 \times \mathrm{pH} 6.5 \odot \mathrm{pH} 7.5+\mathrm{pH} 8.0$

Figure 3 Stern-Volmer plot for erythropoietin at various $\mathrm{pHs}$.

of exposure of the hydrophobic Trp residues. The results also showed that EPO is relatively more stable and resistant than BSA to the effect of $\mathrm{pH}$.

The Stern-Volmer equation was developed without the inclusion of fluorophore concentration as a variable, suggesting that fluorescence quenching is independent of the fluorophore concentration. For a purely collisional quenching mechanism, the Stern-Volmer plot of $F_{0} / F$ vs $[Q]$ would give a linear plot, with $\mathrm{K}_{\mathrm{SV}}$ as the slope. The $\lambda_{\max }$ of EPO remained unchanged at $336 \mathrm{~nm}$ indicating that the Trp residues are located in the highly nonpolar region of the glycoprotein. The $\lambda_{\text {max }}$ remained unchanged even after exposure to $60^{\circ} \mathrm{C}$ for $2 \mathrm{~h}$ (data not shown). A linear Stern-Volmer plot (pH 6.5) suggests that only one class of EPO Trp residues are quenched, and that they are all equally accessible to the quenching of iodide (Figure 3). A nonlinear positive curvature $(\mathrm{pH} 5.0,8.0)$ indicates that two classes of Trp residues are present, one of which is more accessible than the other. Generally, a less sensitive system will have a lesser slope, indicated by lower $\mathrm{K}_{\mathrm{sV}}$ value (Table 1). The $\mathrm{K}_{\mathrm{sV}}$ value for iodide quenching at $\mathrm{pH} 5.0$ was $2.2179,1.5$-fold higher than that of $\mathrm{pH} 6.5$ (1.5398). These results showed that at $\mathrm{pH} 6.5$ and 7.5, the class of Trp residues is largely buried within the glycoprotein structure.

Table I The Stern-Volmer constant $\left(\mathrm{K}_{\mathrm{sv}}\right)$ of erythropoietin at $\mathrm{pH}$ 5.0-8.0

\begin{tabular}{lll}
\hline $\mathbf{p H}$ & $\mathbf{K}_{\mathrm{sv}}$ & Linear regression $\left(\boldsymbol{r}^{2}\right)$ \\
\hline 5.0 & 2.218 & 0.924 \\
5.5 & 1.806 & 0.956 \\
6.5 & 1.540 & 0.971 \\
7.5 & 1.604 & 0.960 \\
8.0 & 1.778 & 0.864 \\
\hline
\end{tabular}

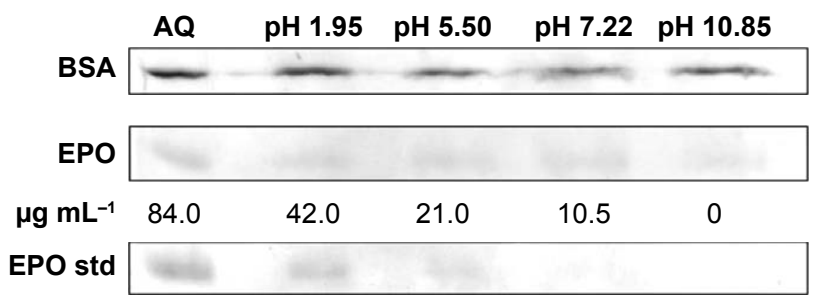

Figure 4 Unbound BSA and EPO in EPO-TAMNLC suspension at various pHs. Abbreviations: BSA, bovine serum albumin; EPO, erythropoietin; TAMNLC, tamoxifen-loaded nanostructured lipid carrier; AQ, aqueous phase; std, standard.

The amount of proteins which did not bind to the proteinTAMNLC complex determined by SDS-PAGE method is shown in Figure 4.

The band intensities of the unbound EPO are shown in Table 2. The result provides a qualitative comparison of binding efficiencies between EPO and TAMNLC at various $\mathrm{pHs}$. There was a good linear correlation between band intensity and concentration of EPO. The aqueous phase (AQ), which was the suspending medium of TAMNLC, served as the negative control. The concentration of unbound EPO in AQ was $41.2 \mu \mathrm{g} \mathrm{mL}^{-1}$ that was almost similar to the initial concentration of $42.0 \mu \mathrm{g} \mathrm{mL}^{-1}$, suggesting that there was no unwanted interaction of EPO in solution to hinder its migration in the gel. The efficiency of association of EPO and TAMNLC was generally $\mathrm{pH}$ dependent, and increased based on the $\mathrm{pH}$ in the following order: $\mathrm{pH} 7.20>\mathrm{pH} 1.91>$ $\mathrm{pH} 5.50>\mathrm{pH} 10.85$.

Figure 5 shows the titration profile of EPO and TAMNLC interaction at $25^{\circ} \mathrm{C}, 30^{\circ} \mathrm{C}$, and $37^{\circ} \mathrm{C}$ where the titration of $2 \mu \mathrm{L}$ of EPO into TAMNLC generated a large amount of binding endothermic heat that gradually decreased in magnitude with each successive titration. The raw heat upon integration was endothermic. The normalized heat of EPO and TAMNLC association could be best fitted with "independent" binding model where their thermodynamic parameters can be extracted. It should be noted that the first titration point was excluded in order to improve the accuracy of the integration and thus the thermodynamic parameters.

The association constant $\left(\mathrm{K}_{\mathrm{a}}\right)$, stoichiometry (n), enthalpy of binding $(\Delta \mathrm{H})$, entropy of binding $(\Delta \mathrm{S})$, and free energy of binding $(\Delta \mathrm{G})$ yielded from the fitting of the binding isotherm to the nonlinear binding model at different temperatures are indicated in Table 3. The values suggested that the binding between EPO and TAMNLC was spontaneous and driven predominantly by entropic factors with positive enthalpy. The enthalpy and entropy generally increased with increasing temperature; however, the enthalpy did not exhibit a clear linear dependency on 
Table 2 The efficiency of binding of EPO to TAMNLC and AQ at various $\mathrm{pHs}$

\begin{tabular}{|c|c|c|c|c|c|}
\hline Sample & Band intensity & Concentration $\left(\mu \mathrm{g} \mathrm{mL}^{-1}\right)$ & Bound EPO & Binding efficiency (\%) & Expected activity (IU) \\
\hline EPO-AQ & 17897132 & 37.87 & 4.13 & 9.84 & 492.11 \\
\hline \multicolumn{6}{|c|}{ EPO-TAMNLC } \\
\hline $\mathrm{pH} \mathrm{I.95}$ & 9182160 & 21.28 & 20.72 & 49.33 & $2,466.57$ \\
\hline $\mathrm{pH} 5.50$ & 9882679 & 22.61 & 19.39 & 46.16 & $2,307.86$ \\
\hline $\mathrm{pH} 7.20$ & 7835736 & 18.72 & 23.28 & 55.43 & $2,771.62$ \\
\hline $\mathrm{pH} 10.85$ & 10640600 & 24.05 & 17.94 & 42.72 & $2,136.15$ \\
\hline
\end{tabular}

Abbreviations: EPO, erythropoietin; TAMNLC, tamoxifen-loaded nanostructured lipid carrier; AQ, aqueous phase.

temperature. Therefore, the heat capacity of the interaction could not be determined.

The titration of TAMNLC with BSA yielded relatively linear isotherms, marked with consistent raw heat data at $25^{\circ} \mathrm{C}, 30^{\circ} \mathrm{C}$, and $37^{\circ} \mathrm{C}$ (Figure 6). The positive enthalpies were smaller than that of the heat generated by the titration of EPO with TAMNLC even after raising the BSA concentration five times greater than the EPO concentration (data not shown). The isotherms of BSA and TAMNLC interaction could not be analyzed satisfactorily with NanoAnalyze software due to fluctuating heat data at magnified scale, suggesting that there is lack of interaction between BSA and TAMNLC.

The presence of ER $\alpha$ and EpoR on MCF-7 and MDAMB-231 cells was demonstrated with immunochemical staining (Figure 7A1-D3). The MCF-7 cell is intensely ERo-positive, while MDA-MB-231 is ERo-negative. EpoR is present in both the MCF-7 and MDA-MB-231 cells. In the MCF-7 cells, EpoR is mostly in the nucleus, while in the MDA-MB-231 cells, it is mainly in the cytoplasm around the nucleus. This indicates that EpoR in MDA-MB-231 cells is sequestered at the nuclear membrane and/or distributed in the cytoplasm.

Except EPO and DMSO, all treatments caused concentration-dependent cytotoxicity to the breast cancer cells. Our data showed that EPO-TAMNLC and TAMNLC had almost similar cytotoxicity profile (Figure 8A). While the concentration-response curve of TAM and EPO-TAM treatment slowly shifted to the left, the curve of EPO-TAMNLC and TAMNLC treatment shifted to the right from 24 to $48 \mathrm{~h}$, suggesting quicker cytotoxic effect of EPO-TAMNLC and TAMNLC. $\mathrm{GI}_{50}$ values were significantly lower $(P<0.05)$ for both the treatments with NLC compared to those without NLC (Figure 8B). Despite no significant difference in the values of the response parameters was observed between all treatments at 48 and $72 \mathrm{~h}$, the lower $\mathrm{GI}_{50}$ values of EPOTAMNLC at $24 \mathrm{~h}$ confirmed the incorporation with carriers produced a significant effect even at an earlier time point.

The HDFa cell lines derived from adult human fibroblasts were used as normal cells to evaluate the selectivity and safety of EPO-TAMNLC. Figure 9 shows the viability of HDFa and MCF-7 cells after treated with EPO-TAMNLC. The EPO-TAMNLC was $\sim 10$-fold more cytotoxic toward MCF-7 cells than HDFa. While TAM exhibited cytotoxic effect on the cancerous cells, the use of nanocarriers did not significantly improve its cytotoxic effect. However, the viability of normal cells when treated with the drug nanocarriers was higher. This means the incorporation of the EPOTAM into NLC dramatically reduced its cytotoxicity toward the normal cells without compromising its selectivity toward the cancerous cell lines.

\section{Discussion}

Cancers are plagued by other disorders including anemia. ${ }^{19}$ Anemia in cancers is not just due to the disease but also a consequence of chemotherapy. ${ }^{20}$ In the treatment of anemia of malignancies, EPO was thought to be safe until the EpoR was identified in various types of cancer cells, including breast cancer cells. The expression of EPO and the EpoRs in cancer cells has generated much interest because of the concern that administration of EPO to patients with cancers expressing the EpoRs may promote tumor growth via the induction of cell proliferation. ${ }^{21}$ However, the role of growth promotion by EPO in tumors expressing EpoRs remains unclear. There are evidences that show that expression of EpoRs on cancer cells is beneficial. Several preclinical studies have shown that EPO delays tumor growth. It is conceivable that increased expression of EPO could reduce tumor hypoxia and ameliorate the deleterious effects of hypoxia on tumor growth, metastasis, and treatment resistance. ${ }^{21,22}$ The expression of EpoRs in cancer cells, coupled with the reported cell growth-promoting ability of EPO, has raised concern that administration of recombinant human EPO to cancer patients with anemia may enhance proliferation and/or survival of

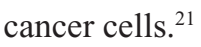

Although EpoRs have been detected in tumor cells, there is no compelling evidence at present that exogenous EPO confers a proliferative effect on the cancer cells. Among these uncertainties, the expression of EpoRs on cancer cells could 
A

Time (s)

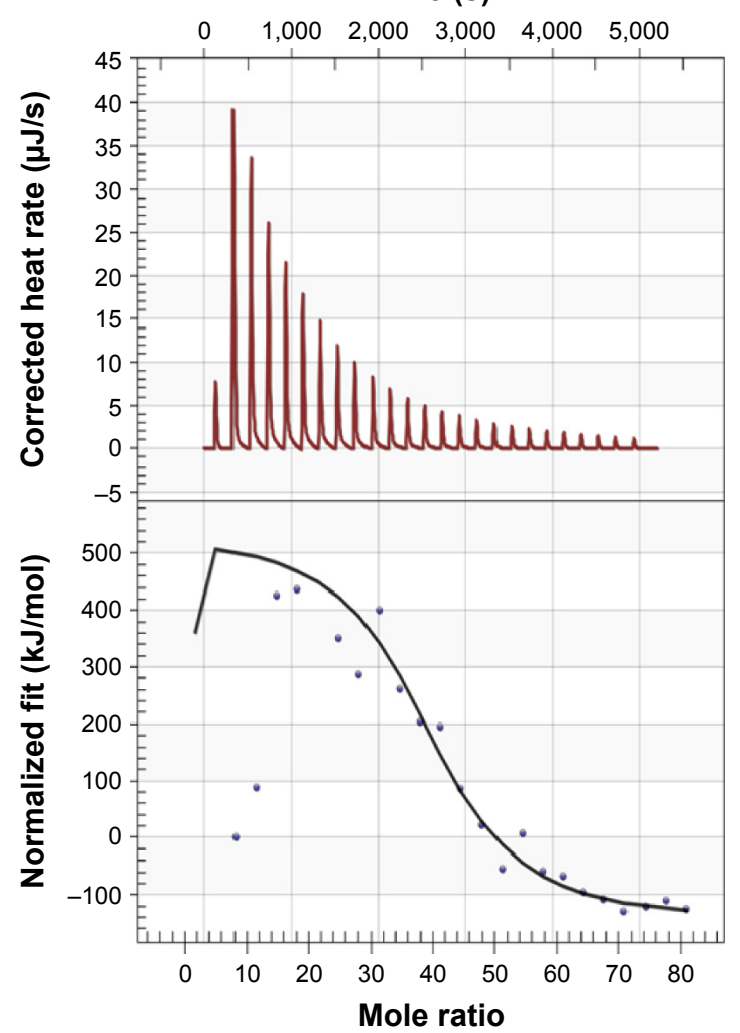

C
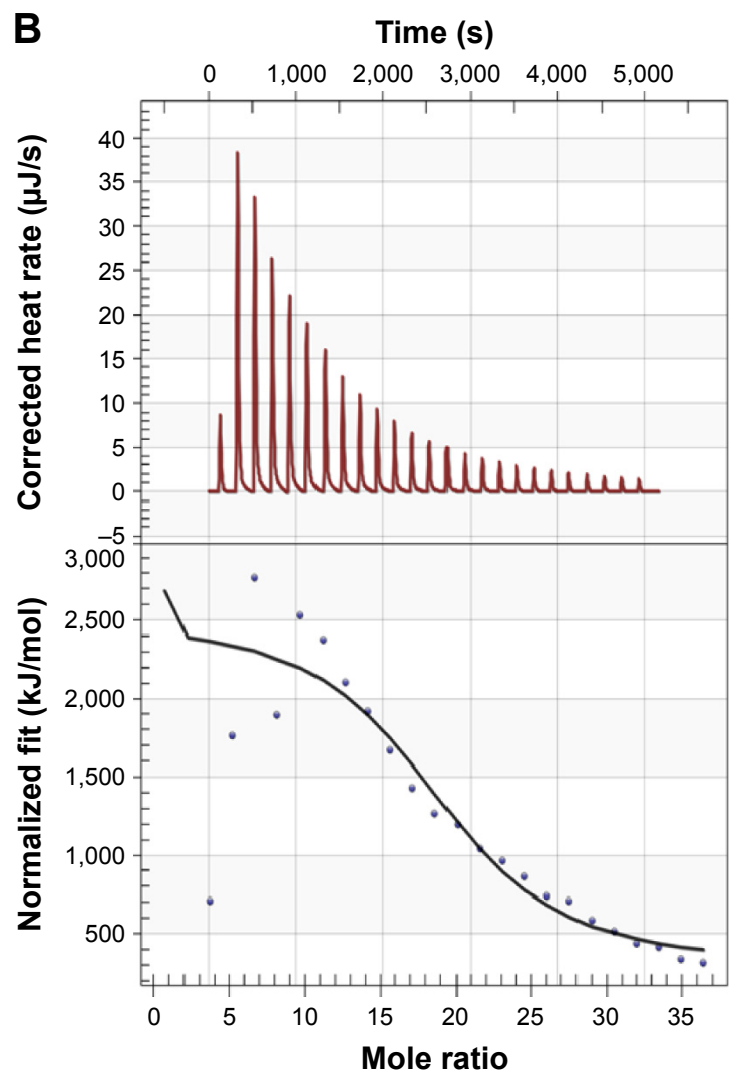

Time (s)

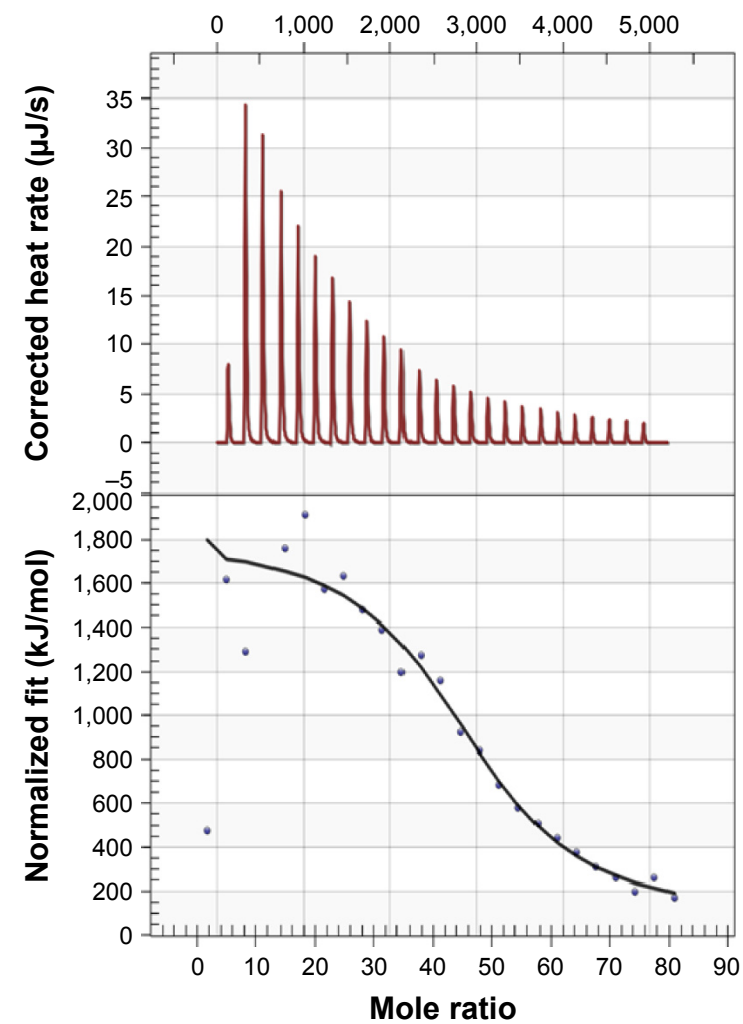

Figure 5 ITC for the association between EPO and TAMNLC at different temperatures. The ITC profiles are shown for the binding of EPO to TAMNLC at (A) $25^{\circ} \mathrm{C}$, (B) $30^{\circ} \mathrm{C}$, and $(\mathbf{C}) 37^{\circ} \mathrm{C}$. The upper panels represent the raw heat after subtracting their respective baselines, while the bottom panels represent the integrated heat released during binding at $25^{\circ} \mathrm{C}, 30^{\circ} \mathrm{C}$, and $37^{\circ} \mathrm{C}$. The solid lines in the lower panels represent the best curve fitted for the experimental data using an independent model. Abbreviations: ITC, isothermal titration calorimetry; EPO, erythropoietin; TAMNLC, tamoxifen-loaded nanostructured lipid carrier. 
Table 3 Thermodynamic parameters for the association of erythropoietin and tamoxifen-loaded nanostructured lipid carriers

\begin{tabular}{|c|c|c|c|c|c|}
\hline Temperature $\left({ }^{\circ} \mathrm{C}\right)$ & $\mathrm{K}_{\mathrm{a}} \times 10^{5}\left(\mathrm{~mol}^{-1}\right)$ & $\mathbf{n}$ & $\Delta \mathrm{H}\left(\mathrm{kJ} \mathrm{mol} \mathrm{mo}^{-1}\right)$ & $\Delta S\left(\mathbf{k J ~ m o l} \mathbf{~}^{-1}\right)$ & $\Delta \mathbf{G}\left(\mathbf{k J ~ m o l} \mathbf{l}^{-1}\right)$ \\
\hline 25 & 215.0 & 39.2 & 690 & 2.46 & -41.59 \\
\hline 30 & 151.4 & 19 & 2,245 & 7.54 & -40.23 \\
\hline 37 & 26,200 & 45 & 2,090 & 6.87 & -40.32 \\
\hline
\end{tabular}

Notes: $\mathrm{K}_{\mathrm{a}}$ is association constant, $\mathrm{n}$ is stoichiometry of binding, $\Delta \mathrm{H}$ is enthalpy of binding, $\Delta \mathrm{S}$ is entropy of binding, and $\Delta \mathrm{G}$ is Gibbs free energy of binding.

be used to advantage. ${ }^{22}$ In fact, the cancer cells membrane EpoRs could be a target for cancer therapy because they do not influence the proliferation of cancer cell lines. ${ }^{23-25}$ In animal models, EPO administration was shown to be beneficial as it increased the tumor sensitivity to cytotoxic drugs, caused tumor regression, improved cytotoxic efficacy, and prolonged survival. ${ }^{26-28}$

TAM is a selective ER modulator and is a most commonly used drug for the treatment of breast cancers. However, treatment with this drug produces numerous side effects. ${ }^{29}$ One way to minimize these side effects is to incorporate the drug in a carrier system that allows slow and prolonged release, while protecting the body system from sudden exposure to the cytotoxic drug. TAM has been successfully incorporated in solid lipid nanoparticles (SLNs) and NLCs. ${ }^{12,30,31}$ The NLC is a second-generation SLN and was shown to be a better carrier for TAM than SLN. ${ }^{15}$ TAMNLC is stable, with good drug-release characteristics, while preserving the anticancer effects of TAM.

The loading of lipid nanoparticles with two therapeutic compounds has not been reported. In this study, the NLC was

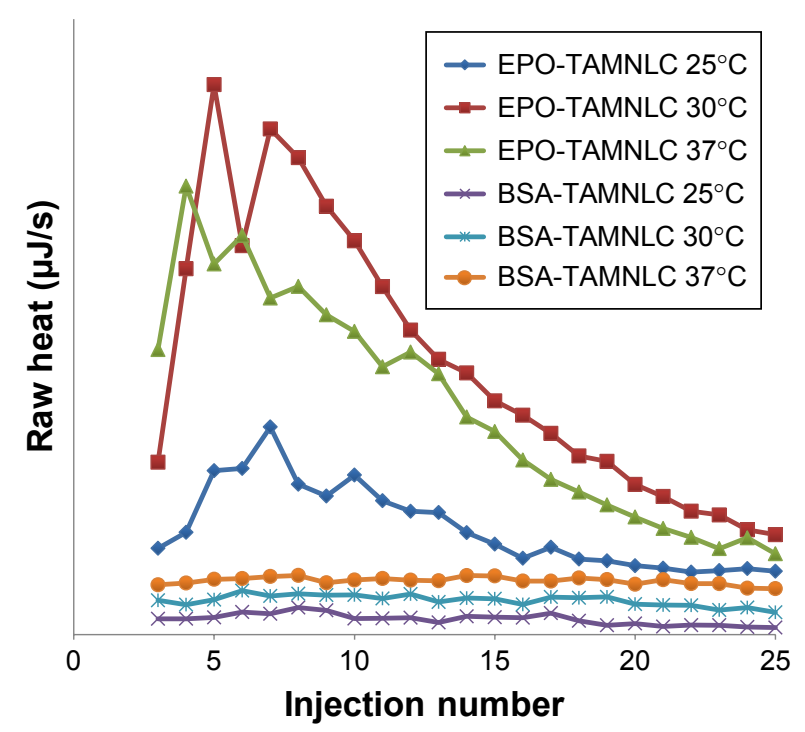

Figure 6 Binding isotherm for the titration of TAMNLC with BSA or EPO. EPOTAMNLC and BSA-TAMNLC complexes are shown at different temperatures. Abbreviations: TAMNLC, tamoxifen-loaded nanostructured lipid carrier; BSA, bovine serum albumin; EPO, erythropoietin. loaded with TAM and coated with EPO. Drug carriers like lipid nanoparticles must be $<100 \mathrm{~nm}$ in diameter to evade the mononuclear phagocyte system (MPS) and $>10 \mathrm{~nm}$ to avoid elimination through glomerular filtration. ${ }^{32}$ The TAMNLC developed in this study with its optimal particle size, narrow polydispersity index, and high entrapment efficiency is suitable as a drug carrier. ${ }^{33}$ With EPO adsorbed on the surface, the changes in the size and polydispersity index of TAMNLC were marginal. However, the EPO increased the zeta potential to a value close to zero, suggesting that the diffused layer around the nanoparticles in solution is thin. The presence of an EPO layer around the TAMNLC masked the electrostatic charge increasing the risk of particle coalescence. However, the surfactant, polysorbate 80 , which was an important component of the NLC, facilitated particle stabilization and significantly reduced the tendency for aggregation. . $^{3435}$

In blood circulation, the nanoparticles are subjected to interactions with serum albumin that may alter their pharmacokinetics and efficacy. ${ }^{36} \mathrm{At} \mathrm{pH} \mathrm{7.4,} \mathrm{which} \mathrm{is} \mathrm{the} \mathrm{average}$ $\mathrm{pH}$ of blood and in high-albumin concentration medium, the stability and aggregability of EPO-TAMNLC were not affected, suggesting that the nanoparticles are suitable for parenteral application. Concentrations of EPO and TAMNLC should be known to estimate the stoichiometry of the interaction. However, due to the lack of an established method for the determination of the concentration of nanocarriers, we proposed a mathematical calculation to measure the concentration of TAMNLC (Supplementary materials). The concentration of TAMNLC, expressed in molarity, is determined based on the specific surface area of the nanoparticle. Using this method, the number of TAMNLC particles can be determined and converted into moles by using the Avogadro's number.

The inability of lipid nanoparticles to interact with serum proteins is a putative factor for their prolonged residence in circulation and biodistribution. This attribute was evident with PEGylated liposomes and conjugated biomolecules. ${ }^{37,38}$ Due to their increased hydrophilic properties, the surface-modified liposomes performed better in preventing aggregations, 

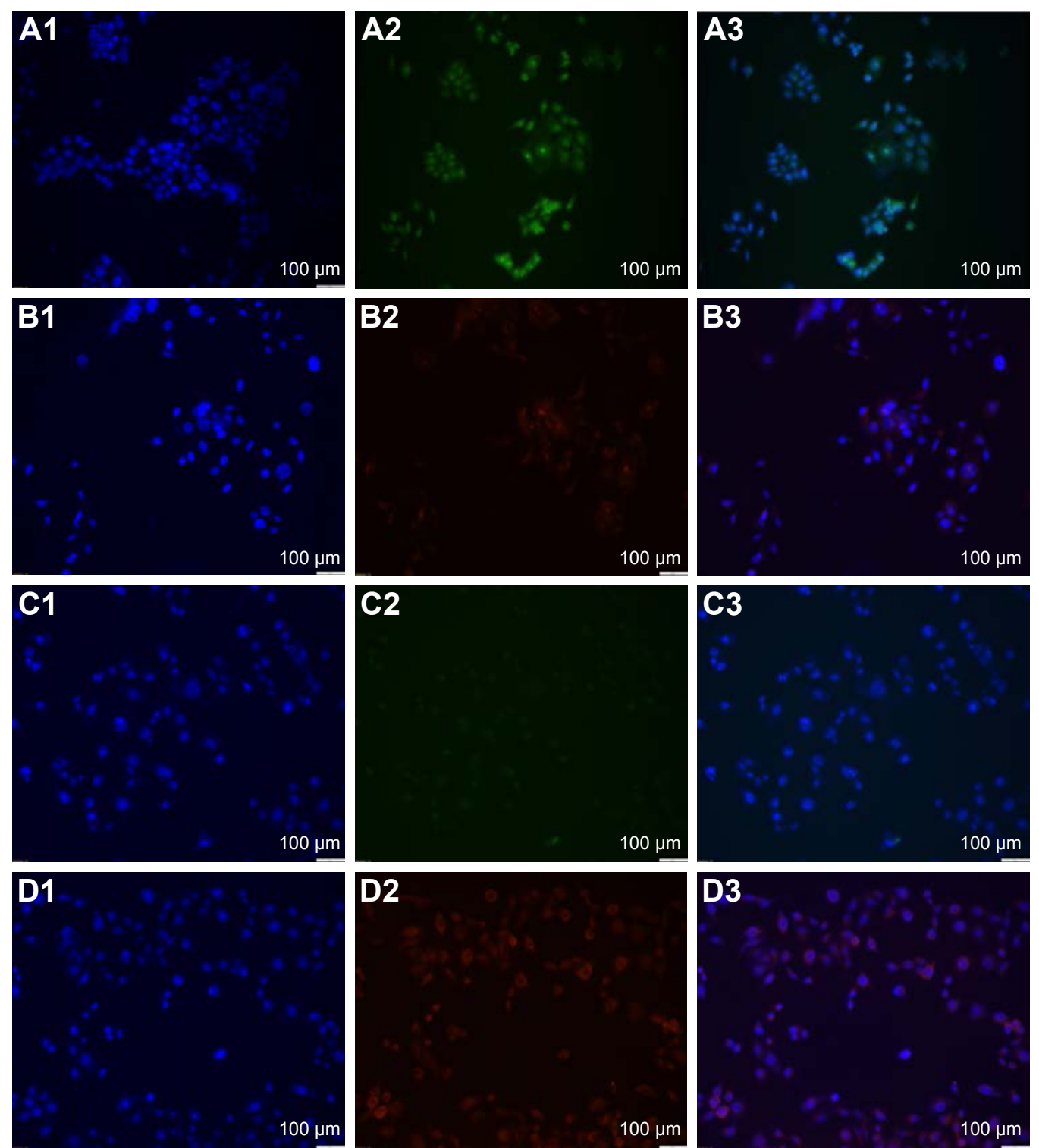

Figure 7 Fluorescence images of ER $\alpha$ and EpoR expressed on (AI-B3) MCF-7 and (CI-D3) MDA-MB-23I cells. Column I: 4',6-diamidino-2-phenylindole stain (blue); column 2: ER $\alpha$ (green) or EpoR (red); and column 3: merged image. (A3) ER $\alpha$ appears within and around the blue nucleus of the MCF-7 cells. Some MCF-7 cells show higher intensity of ER $\alpha$ in the nuclei than the cytoplasm. (C2 and C3) ER $\alpha$ is minimally present in the MDA-MB-23I cells. (B3 and D3) EpoR is present in both the MCF-7 and MDA-MB-23I cells (I00× magnification).

Abbreviations: $\mathrm{ER} \alpha$, estrogen receptor- $\alpha$; EpoR, erythropoietin receptor.

MPS clearance, and elimination by serum proteins, thus prolonging the drug circulation time. Similar effects were observed with polysorbate 80 -coated polybutylcyanoacrylate nanoparticles which exhibited longer circulation time than uncoated nanoparticles. ${ }^{39}$ With polysorbate 80 included in formulation, the EPO-TAMNLC seems to be favorable for clinical application because during intravenous administration, it is less likely for serum albumin to displace EPO in the EPO-TAMNLC. In addition, the high $\mathrm{K}_{\mathrm{a}}$ reflects good binding affinity between EPO and TAMNLC at body temperature. While it is unclear how TAMNLC favors the association with EPO instead of albumin, we hypothesize that the carbohydrate chains on the EPO containing sialic acid residues play a role in the interaction.

Generally, the number of binding sites on TAMNLC increases with temperature. It is presumed that the increase in binding sites is not due to the increased surface area. This is because during the experimental procedure at $37^{\circ} \mathrm{C}$, the heat did not cause significant change in the structure and crystallization of NLC. ${ }^{15}$ Furthermore, aggregation of EPO such as by dimerization will only take place upon storage at $40^{\circ} \mathrm{C}$ for 2 weeks. ${ }^{40}$ These results also indicate that the increase 

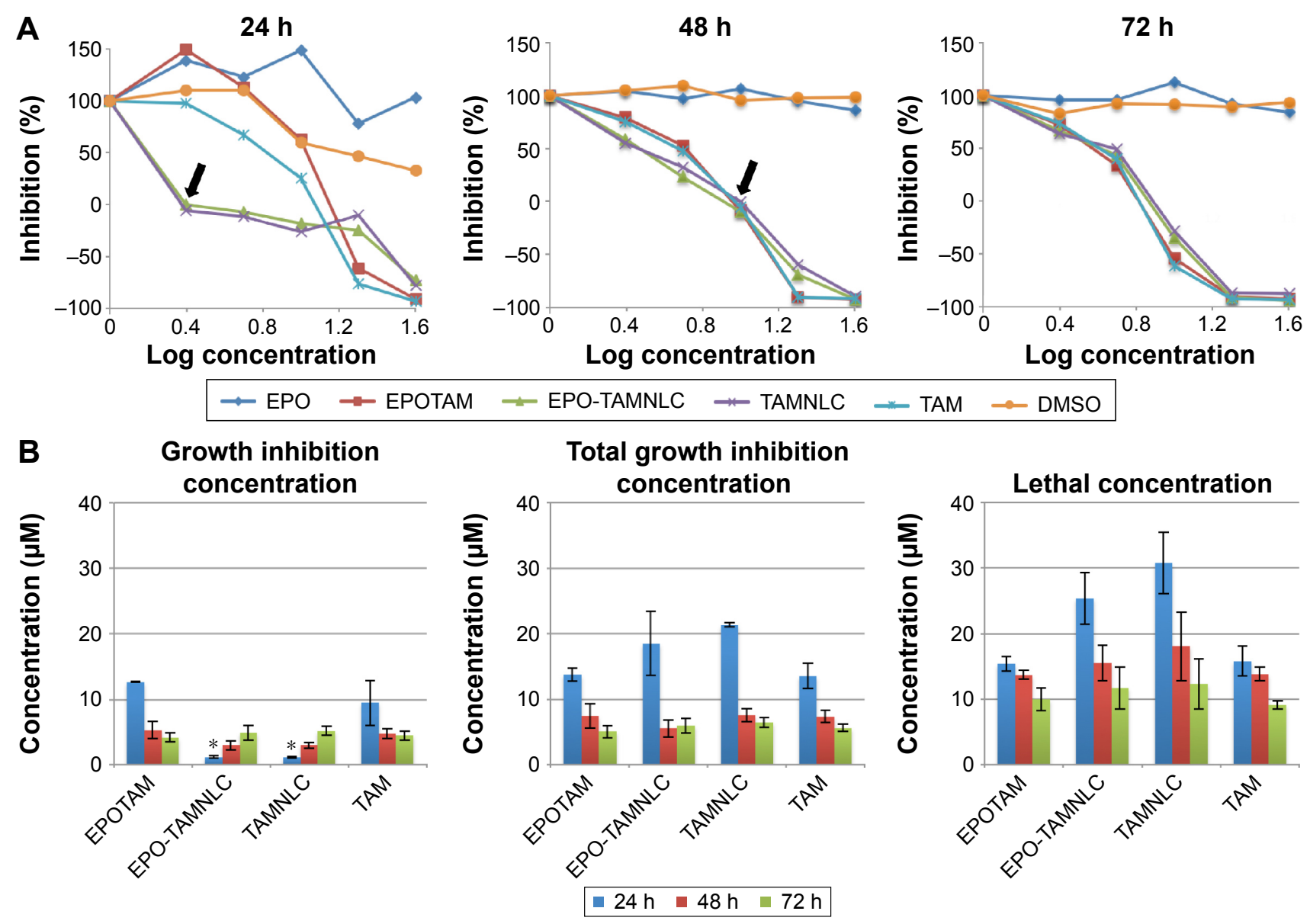

Figure 8 Cytotoxicity of EPO, TAM, EPOTAM, EPO-TAMNLC, TAMNLC, and DMSO treatments on MCF-7 cells. (A) Concentration-response curve of various treatments at $24 \mathrm{~h}, 48 \mathrm{~h}$, and $72 \mathrm{~h}$. The curve shifted to the right in response to time when NLC was used as a carrier for TAMNLC and EPO-TAMNLC (black arrows). (B) Calculated response parameters of EPOTAM, EPO-TAMNLC, TAMNLC, and TAM ( $n=3,{ }^{*} p<0.05$ vs EPOTAM and TAM group).

Abbreviations: EPO, erythropoietin; TAM, tamoxifen; TAMNLC, tamoxifen-loaded nanostructured lipid carrier; DMSO, dimethyl sulfoxide; NLC, nanostructured lipid carrier.

in stoichiometry is likely the result of the binding of EPO to TAMNLC alone. At body temperature, increased exposure of the hydrophobic amino acid residues of EPO molecules may improve the interactions between EPO and TAMNLC. The ITC analysis provides thermodynamic information which

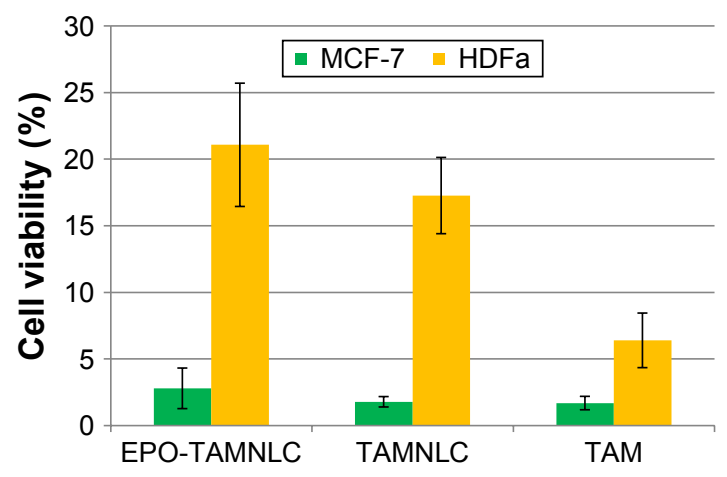

Figure 9 Comparison of cell viability percentage between MCF-7 and HDFa cells for EPO-TAMNLC, TAMNLC, and TAM at $20 \mu \mathrm{M}$ after $72 \mathrm{~h}$ of incubation.

Abbreviations: EPO, erythropoietin; TAMNLC, tamoxifen-loaded nanostructured lipid carrier; TAM, tamoxifen. is relevant not only to conventional drug-drug interaction and enzyme kinetics but also to the development of ligandconjugated nanoparticles such as EPO-TAMNLC.

To achieve active targeting using the EPO-TAMNLC, it is essential to elucidate the status of EpoR in cancerous cells. The significance of the presence and functionality of EpoR on breast cancer cells has been debatable. As such, the relationship between EpoR and ER and efficacy of breast cancer chemotherapy has been investigated. Teo and Rasedee have demonstrated a modulatory effect of EPO on breast cancer cell survival and proliferation, which is ER dependent. ${ }^{41,42}$ They reported the synergistic effect of EPO and TAM that is capable of reducing the clonogenicity of the cancerous cells and cell viability significantly more than TAM alone..$^{43}$ In a separate study, the combination of EPO and TAM resulted in a $90 \%$ tumor regression in mammary tumor-induced Sprague Dawley rats, which was $20 \%$ more than that obtained with TAM alone. ${ }^{8}$ The fact that EpoR is present in tumor cells and absent in the surrounding normal breast tissues 
implies EPO is a potential ligand for site-specific targeting of breast cancers. ${ }^{7}$

We have previously developed the NLC formulation using biocompatible materials and demonstrated its biological inertness via in vitro and in vivo methods. ${ }^{12,33,44}$ The use of lipid nanoparticulate carriers in enhancing the anticancer effect of API has been well documented. ${ }^{45}$ The efficacy of EPO-TAMNLC as an anticancer composition can be attributed to the presence of lecithin and enhanced cellular uptake of the loaded nanoparticle.

It was shown that conjugation of TAM to nanocarriers has an effect on efflux transporters including the multidrug resistance-associated proteins by promoting accumulation of drugs in cancerous cells. ${ }^{45}$ Lecithin was also implicated as an enhancer of percutaneous permeation through its ability to lower the barrier resistance of stratum corneum, courtesy to its mild emulsification properties. These properties could also disrupt the cell membrane integrity and promote the accumulation of APIs in the cell. Thus, the presence of lecithin in the EPO-TAMNLC formulation further helps in the uptake of the nanoparticles. This accounts for the better cytotoxic effect of EPO-TAMNLC at $24 \mathrm{~h}$. At $\mathrm{LC}_{50}$ however, free TAM appeared more effective than EPOTAMNLC. Free TAM is absorbed into cells via diffusion. Since the route of TAM uptake can affect the fate of drug in the cells, difference in biological responses between TAM and EPO-TAMNLC is expected. Unlike the EPO-TAMNLC dispersion, free TAM is poorly water-soluble and necessitates the use of organic solvents to solubilize. However, the inclusion of a noxious organic solvent in a drug composition is detrimental to health and uncommon in clinical practice. Thus, solubilizing TAM by incorporation in EPO-TAMNLC complex is the most practical and effective way to improve the therapeutic effects of TAM.

\section{Conclusion}

The significance of EpoR in tumors has been an issue, particularly in the use of EPO as an adjuvant therapy in cancers. However, EpoR expressed on breast cancer cells is a potential receptor for drug-targeted therapy. The EPOTAMNLC, besides being a depot for TAM, could provide a means for the nanoparticle to bind to the EpoR on cancer cells. The EPO-TAMNLC may not be affected by the serum albumin in circulation. The EPO-TAMNLC could enhance the in vitro cytotoxicity of TAM and MCF-7 cells selectivity. The EPO surface-functionalized TAMNLC while possessing cancer-targeting properties could also deliver EPO and TAM in a sequential manner that may be beneficial to breast cancer patients.

\section{Acknowledgments}

This work was funded by a grant from the Ministry of Science, Technology and Innovation (MOSTI) for a project entitled "Enhancement of the cytotoxic effects of selective estrogen receptor modulators on breast cancer by recombinant human erythropoietin". The authors thank Madam Azyyati Mohd Padzil and Malaysia Genome Institute for their technical support and services.

\section{Disclosure}

The authors report no conflicts of interest in this work.

\section{References}

1. Rahman HS, Rasedee A, Abdul AB, et al. Zerumbone-loaded nanostructured lipid carrier induces G2/M cell cycle arrest and apoptosis via mitochondrial pathway in a human lymphoblastic leukemia cell line. Int J Nanomed. 2014;9:527-538.

2. Groopman JE, Itri LM. Chemotherapy-induced anemia in adults: incidence and treatment. J Natl Cancer Inst. 1999;91(19):1616-1634.

3. Dicato M. Venous thromboembolic events and erythropoiesisstimulating agents: an update. Oncologist. 2008;13(Suppl 3):11-15.

4. Leyland-Jones B; BEST Investigators and Study Group. Breast cancer trial with erythropoietin terminated unexpectedly. Lancet Oncol. 2003;4(8):459-460.

5. Arcasoy MO, Amin K, Karayal AF, et al. Functional significance of erythropoietin receptor expression in breast cancer. Lab Invest. 2002;82(7):911-918.

6. Trost N, Juvan P, Sersa G, Debeljak N. Contrasting effect of recombinant human erythropoietin on breast cancer cell response to cisplatin induced cytotoxicity. Radiol Oncol. 2012;46(3):213-225.

7. Acs G, Acs P, Beckwith SM, et al. Erythropoietin and erythropoietin receptor expression in human cancer. Cancer Res. 2001;61(9): 3561-3565.

8. Sairah AK, Rasedee A, Sheikh O, Rozita R, Al-Haj N. The effects of recombinant human erythropoietin and tamoxifen on growth and angiogenesis of mammary tumor in Sprague-Dawley rat. Am J Pharmacol Toxicol. 2009;4(1):12-16.

9. Beloqui A, Solinís MÁ, Rodríguez-Gascón A, Almeida AJ, Préat V. Nanostructured lipid carriers: promising drug delivery systems for future clinics. Nanomedicine. 2016;12(1):143-161.

10. Ng WK, Yazan LS, Yap LH, Wan Nor Hafiza WAG, How CW, Abdullah R. Thymoquinone-loaded nanostructured lipid carrier exhibited cytotoxicity towards breast cancer cell lines (MDA-MB-231 and MCF-7) and cervical cancer cell lines (HeLa and SiHa). Biomed Res Int. 2015;2015:1-10.

11. Rahman HS, Rasedee A, How CW, et al. Antileukemic effect of zerumbone-loaded nanostructured lipid carrier in WEHI-3B cell-induced murine leukemia model. Int J Nanomedicine. 2015;10:1649-1666.

12. How CW, Rasedee A, Manickam S, Rosli R. Tamoxifen-loaded nanostructured lipid carrier as a drug delivery system: characterization, stability assessment and cytotoxicity. Colloids Surf B Biointerfaces. 2013;112:393-399.

13. Ngo CL, Le QT, Ngo TT, Nguyen DN, Vu MT. Surface modification and functionalization of carbon nanotube with some organic compounds. Adv Nat Sci. 2013;4(3):035017.

14. Anbazhagan V, Sankhala RS, Singh BP, Swamy MJ. Isothermal titration calorimetric studies on the interaction of the major bovine seminal plasma protein, PDC-109 with phospholipid membranes. PLoS One. 2011; 6(10):e25993.

15. How CW, Abdullah R, Abbasalipourkabir R. Physicochemical properties of nanostructured lipid carriers as colloidal carrier system stabilized with polysorbate 20 and polysorbate 80. Afr J Biotechnol. 2011;10(9):1684-1689. 
16. Ward LD, Zhang JG, Checkley G, Preston B, Simpson RJ. Effect of $\mathrm{pH}$ and denaturants on the folding and stability of murine interleukin-6. Protein Sci. 1993;2(8):1291-1300.

17. Huang W, Zhang C. Assembly and characterization of lipid-lipid binding protein particles. J Biotechnol. 2011;154(1):60-67.

18. Davis JM, Arakawa T, Strickland TW, Yphantis DA. Characterization of recombinant human erythropoietin produced in Chinese hamster ovary cells. Biochemistry. 1987;26(9):2633-2638.

19. Knight K, Wade S, Balducci L. Prevalence and outcomes of anemia in cancer: a systematic review of the literature. Am J Med. 2004; 116 Suppl 7A:11S-26S.

20. Tas F, Eralp Y, Basaran M, et al. Anemia in oncology practice: relation to diseases and their therapies. Am J Clin Oncol. 2002;25(4):371-379.

21. Farrell F, Lee A. The erythropoietin receptor and its expression in tumor cells and other tissues. Oncologist. 2004;9 Suppl 5:18-30.

22. Lappin TR, Maxwell AP, Johnston PG. EPO's alter ego: erythropoietin has multiple actions. Stem Cells. 2002;20(6):485-492.

23. Rosti V, Pedrazzoli P, Ponchio L, et al. Effect of recombinant human erythropoietin on hematopoietic and non-hematopoietic malignant cell growth in vitro. Haematologica. 1993;78(4):208-212.

24. Selzer E, Wacheck V, Kodym R, et al. Erythropoietin receptor expression in human melanoma cells. Melanoma Res. 2000;10(5):421-426.

25. Westphal G, Niederberger E, Blum C, et al. Erythropoietin and G-CSF receptors in human tumor cells: expression and aspects regarding functionality. Tumori. 2002;88(2):150-159.

26. Blackwell K, Gascón P, Sigounas G, Jolliffe L. rHuEPO and improved treatment outcomes: potential modes of action. Oncologist. 2004; 9 Suppl 5:41-47.

27. Thews O, Kelleher DK, Vaupel P. Erythropoietin restores the anemiainduced reduction in cyclophosphamide cytotoxicity in rat tumors. Cancer Res. 2001;61(4):1358-1361.

28. Doleschel D, Rix A, Arns S, et al. Erythropoietin improves the accumulation and therapeutic effects of carboplatin by enhancing tumor vascularization and perfusion. Theranostics. 2015;5(8):905-918.

29. Amir E, Seruga B, Niraula S, Carlsson L, Ocaña A. Toxicity of adjuvant endocrine therapy in postmenopausal breast cancer patients: a systematic review and meta-analysis. J Natl Cancer Inst. 2011;103(17): 1299-1309.

30. Abbasalipourkabirreh R, Salehzadeh A, Abdullah R. Cytotoxicity effect of solid lipid nanoparticles on human breast cancer cell lines. Biotechnology. 2011;10(6):528-533.

31. Abbasalipourkabirreh R, Salehzadeh A, Abdullah R. Delivering tamoxifen within solid lipid nanoparticles. Pharm Technol. 2011; 35(4):74-80.

32. Venturoli D, Rippe B. Ficoll and dextran vs. globular proteins as probes for testing glomerular permselectivity: effects of molecular size, shape, charge, and deformability. Am J Physiol Renal Physiol. 2005; 288(4):F605-F613.
33. Peters Jr T. All About Albumin: Biochemistry, Genetics, and Medical Applications. San Diego, CA: Academic Press; 1995.

34. Kronberg B, Dahlman A, Carlfors J, Karlsson J, Artursson P. Preparation and evaluation of sterically stabilized liposomes: colloidal stability, serum stability, macrophage uptake, and toxicity. J Pharm Sci. 1990;79(8):667-671.

35. Kawakami K, Hillery AM. Improving the water solubility of poorly soluble drugs. In: Hillery AM, Park K, editors. Drug Delivery: Fundamentals and Applications. 2nd ed. Boca Raton, FL: CRC Press; 2017.

36. Fasano M, Curry S, Terreno E, et al. The extraordinary ligand binding properties of human serum albumin. IUBMB Life. 2005;57(12):787-796.

37. Ambegia E, Ansell S, Cullis P, Heyes J, Palmer L, MacLachlan I. Stabilized plasmid-lipid particles containing PEG-diacylglycerols exhibit extended circulation lifetimes and tumor selective gene expression. Biochim Biophys Acta. 2005;1669(2):155-163.

38. Iversen F, Yang C, Dagnæs-Hansen F, Schaffert DH, Kjems J, Gao S. Optimized siRNA-PEG conjugates for extended blood circulation and reduced urine excretion in mice. Theranostics. 2013;3(3):201-209.

39. Kreuter J, Ramge P, Petrov V, et al. Direct evidence that polysorbate80 -coated poly(butylcyanoacrylate) nanoparticles deliver drugs to the CNS via specific mechanisms requiring prior binding of drug to the nanoparticles. Pharm Res. 2003;20(3):409-416.

40. Chang S-H, Kim H-J, Kim C-W. Analysis of the structure and stability of erythropoietin by $\mathrm{pH}$ and temperature changes using various LC/MS. Bull Korean Chem Soc. 2013;34(9):2663-2670.

41. Teo GY, Rasedee A. Recombinant human erythropoietin: a novel treatment regime to curb breast cancer proliferation. Paper presented at: Pameran Reka Cipta, Penyelidikan dan Inovasi (PRPI) University Putra Malaysia; August 22-24, 2006; Kuala Lumpur.

42. Teo GY, Rasedee A. Recombinant human erythropoietin modulates the anticancer activity of tamoxifen in breast cancer cells. Paper presented at: Pre-summit Scientific Conference, National Summit on Breast Cancer Education; June 30, 2006; Kuala Lumpur.

43. Rasedee A, Rozita R, Noorjahan BMA, Teo GY. EPOTAM - novel treatment regime to curb breast cancer cell proliferation. Paper presented at: International Exposition of Research and Innovations of Instituitions of Higher Learning (PECIPTA). Organized by Ministry of Higher Education and University of Science Malaysia; August 10-12, 2007; Kuala Lumpur.

44. Rahman HS, Rasedee A, Othman HH, et al. Acute toxicity study of zerumbone-loaded nanostructured lipid carrier on BALB/c mice model. Biomed Res Int. 2014;2014:563930.

45. Kuo YC, Cheng SJ. Brain targeted delivery of carmustine using solid lipid nanoparticles modified with tamoxifen and lactoferrin for antitumor proliferation. Int J Pharm. 2016;499(1-2):10-19.
Drug Design, Development and Therapy

\section{Publish your work in this journal}

Drug Design, Development and Therapy is an international, peerreviewed open-access journal that spans the spectrum of drug design and development through to clinical applications. Clinical outcomes, patient safety, and programs for the development and effective, safe, and sustained use of medicines are the features of the journal, which

\section{Dovepress}

has also been accepted for indexing on PubMed Central. The manuscript management system is completely online and includes a very quick and fair peer-review system, which is all easy to use. Visit http://www.dovepress.com/testimonials.php to read real quotes from published authors. 